\title{
The Role of Gonadal Hormones in Neuromuscular Synapse Elimination in Rats. I. Androgen Delays the Loss of Multiple Innervation in the Levator Ani Muscle
}

\author{
Cynthia L. Jordan, Michael S. Letinsky, ${ }^{1}$ and Arthur P. Arnold \\ Department of Psychology and Laboratory of Neuroendocrinology, Brain Research Institute, University of California, Los \\ Angeles, Los Angeles, California 90024, and 'Department of Physiology and Ahmanson Laboratory of Neurobiology, \\ University of California, Los Angeles, Los Angeles, California 90024
}

\begin{abstract}
The normal period of synapse elimination in the androgensensitive levator ani (LA) muscle occurs between 2 and 4 weeks after birth, well after the period of synapse elimination for most other rat muscles. To evaluate whether gonadal androgen might be involved in the delayed development of single innervation in the LA, we compared the time course of synapse elimination in LA muscles that lacked endogenous gonadal androgen or were exposed to exogenous androgen. Tetranitroblue tetrazolium was used to stain neuromuscular connections. Our results suggest that both endogenous and exogenous androgen delay the normal process of synapse elimination. Removing endogenous androgen resulted in lower levels of multiple innervation in the LA, suggesting that androgen may normally influence synapse elimination. Moreover, androgen treatment prevented much of the normal loss of multiple innervation in the LA. Androgen treatment during the normal period of synapse elimination also increased the diameter of LA muscle fibers, enhanced the development of preterminal branching, and increased the number of junctional sites on some LA fibers. Because androgen did not appear to induce the formation of new synapses through sprouting, we conclude that androgen maintained multiple innervation in the LA by preventing the normal ontogenetic process of synapse elimination.
\end{abstract}

Synapse elimination in mammalian muscle involves the transition from a neonatal pattern of multiple innervation to the adult pattern of single innervation (Thompson, 1986). In rats, neuromuscular synapse elimination has generally been found to occur around the first 2 weeks after birth (Bennett and Pettigrew, 1974; Brown et al., 1976; Riley, 1977; Betz et al., 1979; Dennis et al., 1981; Steinbach, 1981; Van Essen, 1982; Balice-Gordon and Thompson, 1988). However, we have shown that in the androgen-sensitive levator ani (LA), a perineal muscle normally present postnatally only in male rats (Wainman and Shipounoff, 1941; Cihak et al., 1970), synapse elimination occurs substantially later (Jordan et al., 1988). This process involves 2 phases in the LA, a period of rapid synapse loss between 14 and $28 \mathrm{~d}$ after birth when the majority of fibers become singly

\footnotetext{
Received Apr. 1, 1988; revised June 9, 1988; accepted June 29, 1988.

We wish to thank Dr. Alan Grinnell for his comments on this manuscript. This work was supported by USPHS Grants HD15021, NS13470, and HD7228.

Correspondence should be addressed to Cynthia L. Jordan, Department of Psychology, University of California, Los Angeles, Los Angeles, CA 90024-1563.

Copyright (C) 1989 Society for Neuroscience $0270-6474 / 89 / 010229-10 \$ 02.00 / 0$
}

innervated, followed by a slower phase that begins after the end of the second postnatal month. Some multiple innervation may persist in the adult LA even following this slower phase of synapse elimination. One of the features that distinguishes the LA musclc from most othcr rat skcletal muscles is its marked sensitivity to androgen (Wainman and Shipounoff, 1941; Venable, 1966; Buresova and Gutmann, 1971; Tucek et al., 1976; Vyskocil and Gutmann, 1977; Souccar et al., 1982). We therefore asked whether gonadal androgen might be involved in regulating synapse elimination in the LA. Although the level of testosterone is much lower in males during juvenile development than in adulthood (Corpechot et al., 1981), this low level of testosterone might nevertheless be responsible for the delayed development of LA innervation.

We assessed the effects of both endogenous and exogenous androgen on synapse elimination in 2 muscles, the androgensensitive LA and the extensor digitorum longus (EDL), a muscle considered to be relatively insensitive to androgen (Vyskocil and Gutmann, 1977; Souccar et al., 1982). The process of synapse elimination was assessed by counting the number of stained motor axons that synapsed on individual muscle fibers. Our results show that both endogenous and exogenous androgen delay the loss of multiple innervation in the LA muscle but not in the EDL. Androgen also appears to spare multiple innervation without causing motor terminals to sprout but does influence a number of other aspects of neuromuscular development.

\section{Materials and Methods}

Neonatal male Sprague-Dawley rats were obtained from pregnant rats received from Simonsen Laboratories about $11 / 2$ weeks prior to parturition. On the day of birth, which was taken to be the first day of postnatal life (P1), the number and sex of rat pups were determined, and females were culled when necessary so that litters contained no more than 8 pups. On P7, male rats were castrated under ether anesthesia. Beginning on P7, castrated rats received daily subcutaneous injections of testosterone propionate (TP) (100 $\mu \mathrm{g} \mathrm{TP} / 50 \mathrm{gm}$ body weight) dissolved in sesame oil or control injections of the vehicle oil. Androgen treatment to prepubertal males was done to mimic the much higher level of endogenous androgen that is normally present in adult males (Smith et al., 1978; Corpechot et al., 1981). The dose of TP was adjusted for only every $50 \mathrm{gm}$ increment in body weight (animals weighing $50 \mathrm{gm}$ or less received $100 \mu \mathrm{g} \mathrm{TP}$, animals weighing 51-100 gm received $200 \mu \mathrm{g}$ TP). Animals generally reached 50 gm by P21. Animals were sacrificed after 1,2 , and 3 weeks of treatment (at P14, 21, and 28) and were compared to normal, untreated males of the same age. Animals killed at P28 were weaned at P21 and housed in group cages until they were killed. Males from at least 2 different litters contributed to each experimental group.

Animals were overdosed with sodium pentobarbital, and the LA and EDL muscles from each animal were dissected and pinned as flat whole 


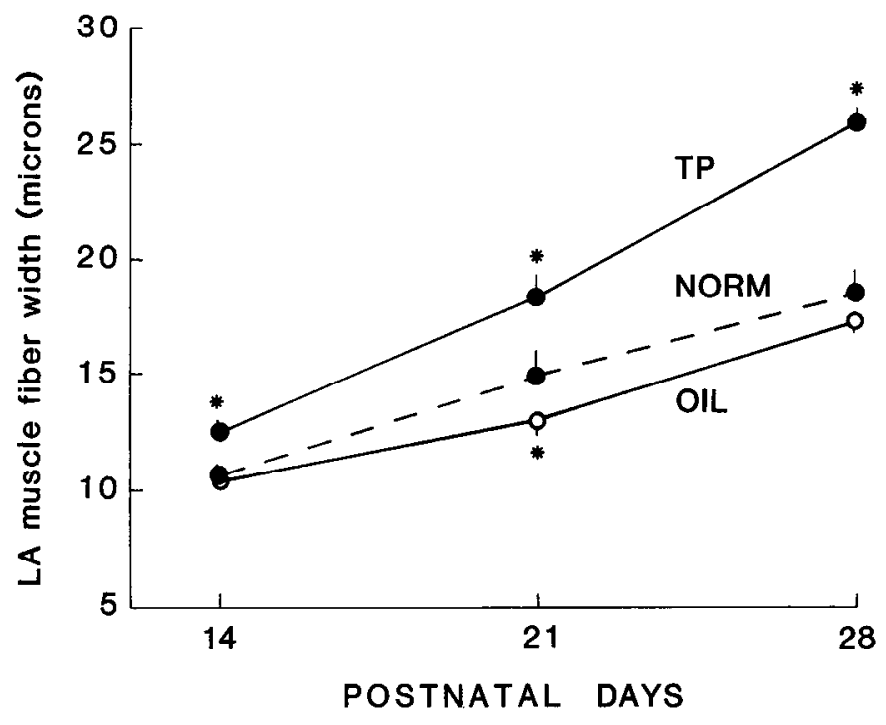

Figure 1. Effect of androgen on LA muscle fiber growth. Each data point represents the mean ( \pm SEM) based on muscles from 5-7 animals per group. $T P, O I L$, and $N O R M$ indicate muscles from androgen-treated castrates, oil-treated castrates, and normal males, respectively. Asterisks indicate significant differences from normal $(p<0.05)$.

mounts in Sylgard-coated dishes containing mammalian Ringer's. The LA was exposed to collagenase [10-30 $\mathrm{min}$ in $2 \mathrm{mg}$ type IV or VII collagenase (Sigma)/ml Ringer's] before staining. Muscles were stained using a modified tetranitroblue tetrazolium (TNBT) protocol and prepared for microscopy as previously described (Jordan et al., 1988).

Stained muscles (from 5-7 animals per group) for each individual age were analyzed as a group without knowledgc of the trcatment condition. We sampled muscle fibers across the entire stained surface of the muscle and included only those fibers that had fully visible nerve terminals and were innervated by unbroken axons that could be followed back into the intramuscular nerves. This sampling criterion was used to increase the probability that individual motor axons that converged on the same muscle fiber were not branches from the same parent motor axon. Although motor axons were seen to branch in the intramuscular nerves, they were never seen to innervate the same fiber. A total of 21-80 muscle fibers per muscle were sampled in this manner (mean number of sampled muscle fibers per age: 28 at P14, 47 at P21, and 48 at P28). We measured the following: (1) the number of apparently separate axons and/or preterminal branches from the same axon that contacted individual fibers; (2) the number of, and distance between, junctional sites on a fiber; (3) the number and length of terminal sprouts; (4) the number of retraction bulbs near but not in contact with a fiber; and (5) the width of individual fibers. These measures are described further under Results. A calibrated ocular micrometer was used for measuring linear distances. Data were statistically analyzed using an analysis of variance (ANOVA) design (Keppel, 1982). A separate analysis was done for each measure in each muscle (LA or EDL). Two-way ANOVAs (treatment $\times$ age) were done unless otherwise specified. Some of the data obtained from normal males is reported in a related study (Jordan et al., 1988).

\section{Results}

Muscle fiber growth

Between P14 and 28, the diameter of LA muscle fibers increased significantly in all groups ( $p$ 's $<0.001$, Fig. 1); LA fiber diameter was also influenced by treatment. LA fibers were significantly larger than normal in androgen-treated muscles at all ages ( $p<0.05$ at P14 and $p<0.001$ at P21 and 28, Fig. 1). However, only at P21 were LA fibers in muscles from oil-treated castrates (which lacked endogenous gonadal androgen) smaller than normal ( $p<0.04$, Fig. 1). The small effect of castration is consistent with the reported low level of endogenous androgen in normal males during the first postnatal month (Corpechot et al., 1981). The size of EDL fibers at P14 averaged about $15 \mu \mathrm{m}$ and did not differ among treatment groups $(p>0.05$, 1-way ANOVA). Since androgen did not influence the level of multiple innervation in the EDL at any age (see below), nor the size of EDL fibers at P14, we did not evaluate the effect of androgen on EDL fiber size at other ages.

\section{Number of axons innervating $L A$ and EDL muscle fibers}

The percentage of multiply innervated muscle fibers was derived from counts of the number of apparently different motor axons that contacted individual muscle fibers. Between P14 and 28, the amount of multiple innervation dropped precipitously in normal LA muscles (Fig. 2; Jordan et al., 1988). In contrast, androgen treatment during this period prevented much of the normal decline of multiple innervation in the LA (Fig. 2). At the earliest age examined, P14, androgen-treated muscles had the same high level of multiple innervation as was present in normal LA muscles $(p>0.05)$. Moreover, at P14 the percentage of LA fibers innervated by 2 versus 3 or more axons also did not differ between androgen-treated and normal muscles $(p>$ 0.05 , Table 1). However, by P21 and 28, androgen-treated LA muscles had significantly more multiply innervated muscle fibers than normal ( $p<0.001$ for both ages). At P21, this difference was present in both the number of fibers innervated by 2 axons $(p<0.003)$ and by 3 or more axons $(p<0.001$, Table 1), whereas at P28, androgen-treated muscles had only significantly more dual innervation than normal $(p<0.001$, Table 1). Conversely, at P14, the incidence of LA fibers innervated by 3 or more axons was significantly less than normal in muscles from oil-treated castrates (with reduced levels of endogenous androgen) $(p<0.001$, Table 1$)$, which accounted for the overall lower level of multiple innervation in these muscles compared with normal ( $p<0.013$, Fig. 2). However, this difference was no longer significant by P21 $(p>0.05)$.

The percentage of multiply innervated fibers in EDL muscles at P14 was unaffected by treatment [ $32 \pm 0.5 \%$ (mean \pm SEM) fibers multiply innervated in normals; $38 \pm 3.8 \%$ in oil-treated castrates and $39 \pm 1.9 \%$ in androgen-treated castrates, $p>0.05$ ]. Because the EDL is normally singly innervated by $P 21$, muscles from castrates were scanned for multiple innervation at $\mathrm{P} 21$ and 28 without rigorous quantification and were found to appear normal and singly innervated.

\section{Frequency of retraction bulbs}

Fine-caliber axons ending in bulbous expansions are present in muscles during the period of synapse elimination (shown schematically in Fig. 3; Riley, 1977, 1981; Jordan et al., 1988). Axons showing such morphology have been called retraction bulbs and are thought to represent retracting axons that once innervated the neuromuscular junction (Riley, 1977, 1981). We estimated the frequency of such retraction bulbs, counting only those found within a few microns of other morphologically intact motor terminals, and excluding those that were present in the intramuscular nerves or in contact with a muscle fiber through a fine interconnective. No more than one retraction bulb was ever found per individual fiber.

At $\mathrm{P} 21$ and 28, retraction bulbs were present at a relatively low incidence (1-6\% of fibers) in LA muscles from androgenand oil-treated castrates. Retraction bulbs were never observed in these muscles at P14, indicating that the time course of appearance of retraction bulbs in experimental muscles is the same as in normal LA muscles (Jordan et al., 1988). Between P21 


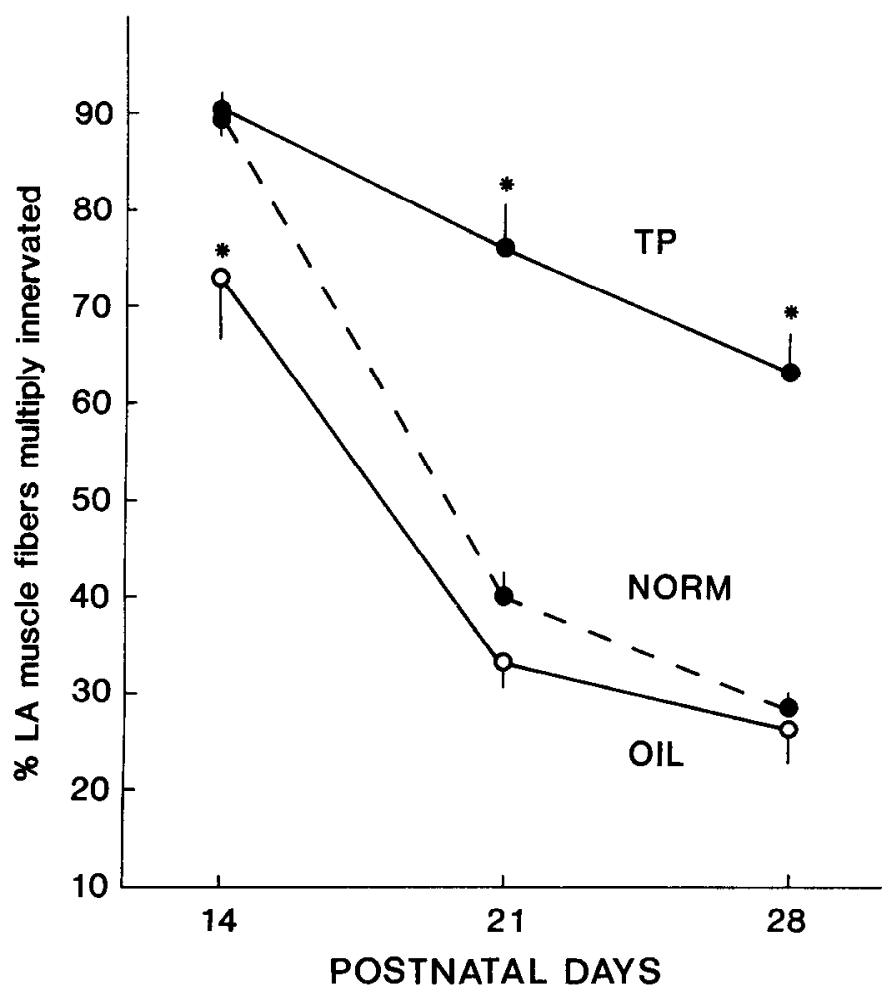

Figure 2. Effect of androgen on the percentage of multiply innervated LA muscle fibers during the normal period of synapse elimination in the LA. Labels are defined as in Figure 1. Asterisks indicate significant differences from normal $(p<0.05)$. Both exogenous and endogenous androgen influence the normal time course of synapse elimination in the LA.

and 28 , the incidence of retraction bulbs declined significantly $(p<0.028)$. Although treatment appeared to influence the incidence of retraction bulbs (Fig. 4), with androgen-treated muscles having fewer and oil-treated muscles having more retraction bulbs than normal at P21, these differences were not significant $(p>0.05$ at either age).

\section{Number of junctional sites per fiber}

In androgen-treated muscles, some of the multiple innervation was in the form of multiple junctional sites (Figs. $5, b, d ; 6$ ), in that multiple axons on some fibers formed distinctly separate terminal arborizations, usually within a few microns of each other. This is in contrast to the morphology of multiple innervation on most LA fibers in muscles from normal males and oil-treated castrates, which involves single junctional sites (Fig. $5, a, c)$. To document this unusual multiple junctional site morphology, we defined a single junctional site as having a presyn-

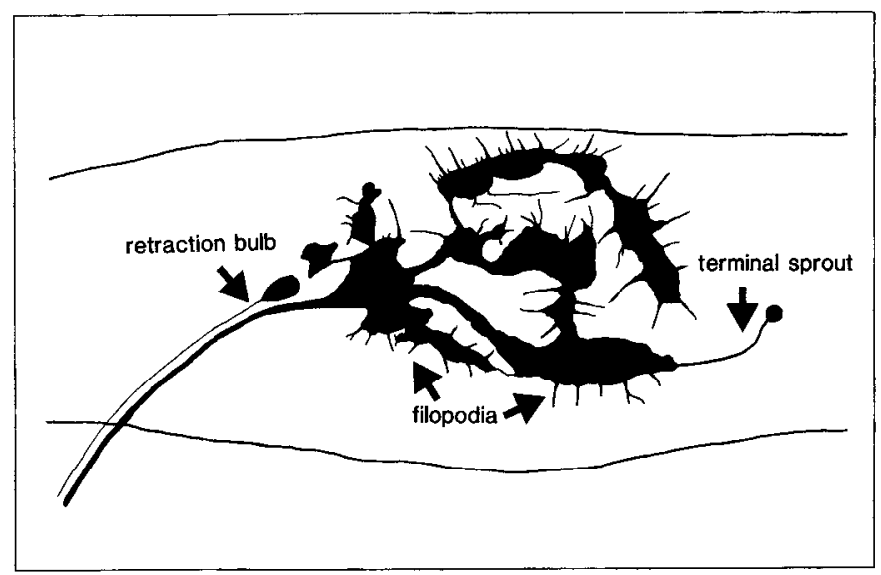

Figure 3. Schematic drawing of a developing LA motor terminal at P21 showing the typical morphology of a retraction bulb, terminal sprout, and filopodium. Retraction bulbs have distinct bulbous endings that are generally formed by relatively small-caliber axons. Filopodia are distinct from terminal sprouts in that they tend to be shorter and finer than terminal sprouts and they appear to taper distally along their length. Terminal sprouts were generally of uniform thickness along their length except for their bulbous endings. Filopodia are transient morphological features of developing motor terminals (Jordan et al., 1988). They are most prominent in the LA at P14, when motor terminals have a growth cone appearance.

aptic arbor whose boundary is circumscribed and completely separate from other presynaptic structures innervating the same muscle fiber. It is important to note that while multiple junctional sites were defined on the basis of an apparent spacing of motor terminals, there did not appear to be any strict rules governing how multiple axons were apportioned among different sites on the same fiber. Individual junctional sites were either singly or multiply innervated and were even occasionally seen to be formed by a preterminal branch from an axon that innervated other sites on the same multiply innervated fiber. For example, at P28 in androgen-treated muscles, of those LA fibers that were multiply innervated and possessed multiple junctional sites (Fig. 6), nearly half had one or more individual sites that were themselves multiply innervated. Moreover, some singly innervated fibers in androgen-treated muscles had multiple junctional sites that obviously were innervated by preterminal branches from the same axon. Presented in the next 3 subsections are quantitative descriptions of (1) the percentage of multiply innervated fibers having multiple junctional sites, (2) the percentage of singly innervated fibers having multiple junctional sites and (3) the average distance between such multiple junctional sites.

Multisite multiple innervation. In androgen-treated LA muscles, multiple junctional sites developed during the time that

Table 1. The percentage of LA muscle fibers innervated by 2 or by 3 or more axons

\begin{tabular}{llllllllr} 
& \multicolumn{2}{l}{ 14 Postnatal days } & & \multicolumn{2}{l}{ 21 Postnatal days } & & & \multicolumn{2}{l}{ 28 Postnatal days } \\
\cline { 2 - 3 } Treatment group & 2 axons & $\geq 3$ axons & & 2 axons & $\geq 3$ axons & & 2 axons & $\geq 3$ axons \\
\hline Normal males & $36 \pm 3.1$ & $53 \pm 3.9$ & & $39 \pm 1.9$ & $2.2 \pm 0.8$ & & $26 \pm 2.2$ & $2 \pm 0.6$ \\
Oil-treated castrates & $49 \pm 3.5$ & $24 \pm 4.8^{a}$ & & $32 \pm 1.6$ & $1.2 \pm 0.8$ & & $26 \pm 3.1$ & $0.2 \pm 0.2$ \\
TP-treated castrates & $41 \pm 2.6$ & $49 \pm 3.5$ & & $55 \pm 5.2^{b}$ & $22 \pm 2.6^{a}$ & & $55 \pm 3.7^{a}$ & $9 \pm 2.1$
\end{tabular}

a Significantly different from normal $(p<0.001)$.

${ }^{b}$ Significantly different from normal $(p<0.01)$. 


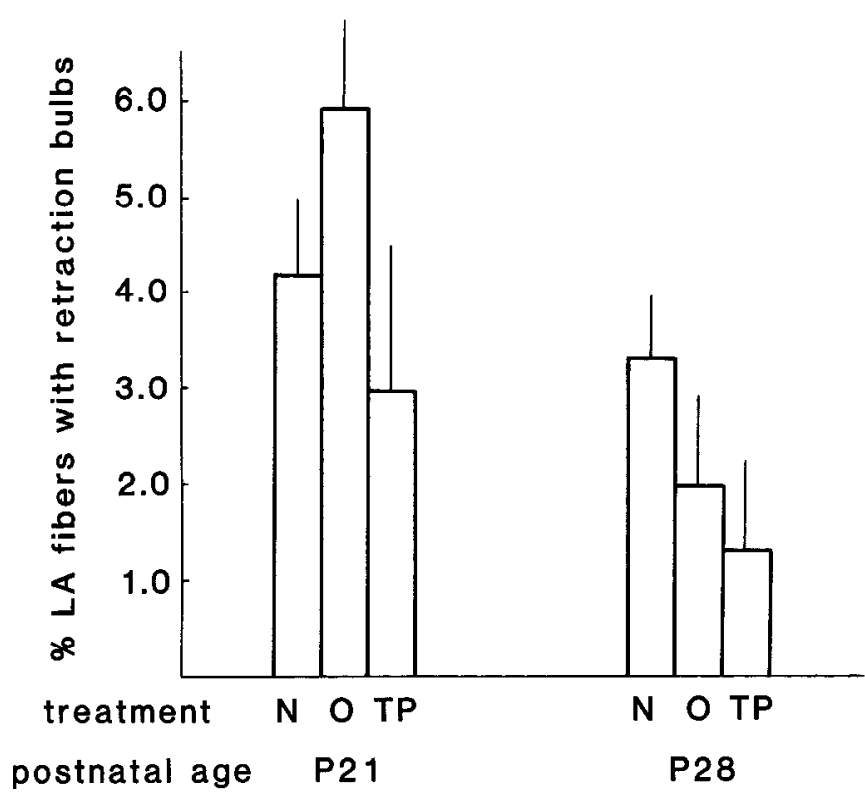

Figure 4. Effect of androgen on the percentage of LA muscle fibers with retraction bulbs in the vicinity of an innervated junction. The height of each bar represents the mean ( \pm SEM) based on muscles from 5-7 animals per group. $N, O$, and $T P$ denote LA muscles from normal males, oil-treated castrates, and androgen-treated castrates, respectively. Retraction bulbs were not detected in I.A muscles at P14.

multiple innervation is normally eliminated (Fig. 6). At P14, most of the multiple innervation in androgen-treated LA muscles was associated with single junctional sites (Fig. 6). At this age, the level of multisite multiple innervation present in androgen-treated muscles was comparable to that found in LA muscles from normals and oil-treated castrates (Fig. 6). By P21 and 28 , however, such multiple junctional sites had developed on 13 and $21 \%$, respectively, of $\mathrm{L} \Lambda$ fibers so that androgentreated muscles at these ages had significantly more multiply innervated fibers of both types than normal (single junctional sites: $p<0.001$ at P21 and $p<0.009$ at P28; multiple junctional sites: $p<0.002$ at $\mathrm{P} 21$ and $p<0.001$ at $\mathrm{P} 28$ ).

Multisite single innervation. More than $99 \%$ of singly innervated fibers in normals and oil-treated castrates at P14, 21, and 28 had single junctional sites. This situation also applies to androgen-treated muscles at P14 and 21. However, by P28, a significant number of singly innervated fibers in androgen-treated muscles had multiple junctional sites $[9.6 \pm 2.4 \%$ ( \pm SEM) of total fibers compared to $1.1 \pm 0.8 \%$ in normals, $p<0.001$ ], indicating that the androgenic induction of multiple junctional sites is not exclusively related to androgen's effect on polyneuronal innervation.

Distance between multiple junctional sites. In the normal LA, there is a small population (about 3\%) of multiply innervated fibers that have 2 separate junctional sites (Jordan et al., 1988). Such normally occurring multiple junctional sites are generally separated by more than $10 \mu \mathrm{m}$. This is in contrast to the extremely close spacing of most junctions on multisite LA fibers in androgen-treated muscles. When multisite fibers are subdivided according to whether the junctional sites were separated by more or less than $10 \mu \mathrm{m}$ (Table 2), it was found that androgen treatment increased only the incidence of fibers having closely spaced sites and not the incidence of fibers with more distantly spaced sites.

\section{Incidence of preterminal branching}

Because LA axons develop preterminal branches during the period of synapse elimination (Jordan et al., 1988), we also assessed the effect of androgen on the development of preterminal branching. Such preterminal branching occurred once an axon had left the intramuscular nerve, generally within $10 \mu \mathrm{m}$ of the motor ending and gave rise to axon branches that innervated the same fiber (Fig. 5). The data on preterminal branching in the LA is presented in 2 ways: (1) the percentage of LA fibers innervated by preterminal branches (Fig. 7) and (2) the average number of preterminal branches that innervated LA fibers (Table 3), a measure that included the total number of axonal inputs (including branches) to a fiber minus the number of apparently different parent axons to that fiber. For example, a fiber innervated by 2 branches of the same axon was considered to have one preterminal branch. This method of estimating the number of preterminal branches was chosen to avoid confounding this measure with the number of different axons that innervated a fiber.

During synapse elimination (P14-28), there was a steady and significant increase in the percentage of LA fibers that were innervated by preterminal branches (Fig. 7). Androgen treatment markedly enhanced the development of preterminal branching during this period. Although significant group differences were not evident at Pl4 $(p>0.05)$, by P21 and 28, androgen treatment significantly increased the incidence of LA fibers innervated by axons that branched preterminally $(p<$ 0.001 at each age; Fig. 7). Further breakdown of the data revealed that androgen increased only the percentage of multiply innervated fibers with preterminal branching $(p<0.001$ at both P21 and P28; Fig. 7) and not the percentage of singly innervated fibers with preterminal branching. However, the apparent lack

Table 2. Total percentage of LA muscle fibers that have multiple junctional sites, with either closely spaced $(<10 \mu \mathrm{m})$ or more distantly spaced $(\geq 10 \mu \mathrm{m})$ junctions (androgen induced the formation of closely spaced junctions)

\begin{tabular}{lccclll} 
& \multicolumn{2}{l}{21 Postnatal days } & & \multicolumn{2}{l}{28 Postnatal days } \\
\cline { 2 - 3 } \cline { 5 - 6 } Treatment group & $<10 \mu \mathrm{m}$ apart & $\geq 10 \mu \mathrm{m}$ apart & & $<10 \mu \mathrm{m}$ apart & $\geq 10 \mu \mathrm{m}$ apart \\
\hline Normal males & $0 \pm 0$ & $3 \pm 1.29$ & & $0.93 \pm 0.93$ & $1.06 \pm 0.76$ \\
$\quad$ Mean distance apart) & $(0 \mu \mathrm{m})$ & $(33.6 \mu \mathrm{m})$ & & $(7.2 \mu \mathrm{m})$ & $(49.6 \mu \mathrm{m})$ \\
Oil-treated castrates & $1.4 \pm 0.98$ & $0.20 \pm 0.20$ & & $1.47 \pm 0.93$ & $0.32 \pm 0.32$ \\
(Mean distance apart) & $(3.45 \mu \mathrm{m})$ & $(25.6 \mu \mathrm{m})$ & & $(2.4 \mu \mathrm{m})$ & $(38.4 \mu \mathrm{m})$ \\
TP-treated castrates & $12.83 \pm 3.10$ & $3.5 \pm 1.71$ & & $28.4 \pm 4.5$ & $2.93 \pm 0.93$ \\
(Mean distance apart) & $(3.52 \mu \mathrm{m})$ & $(28.7 \mu \mathrm{m})$ & & $(4.3 \mu \mathrm{m})$ & $(36.96 \mu \mathrm{m})$ \\
\hline
\end{tabular}



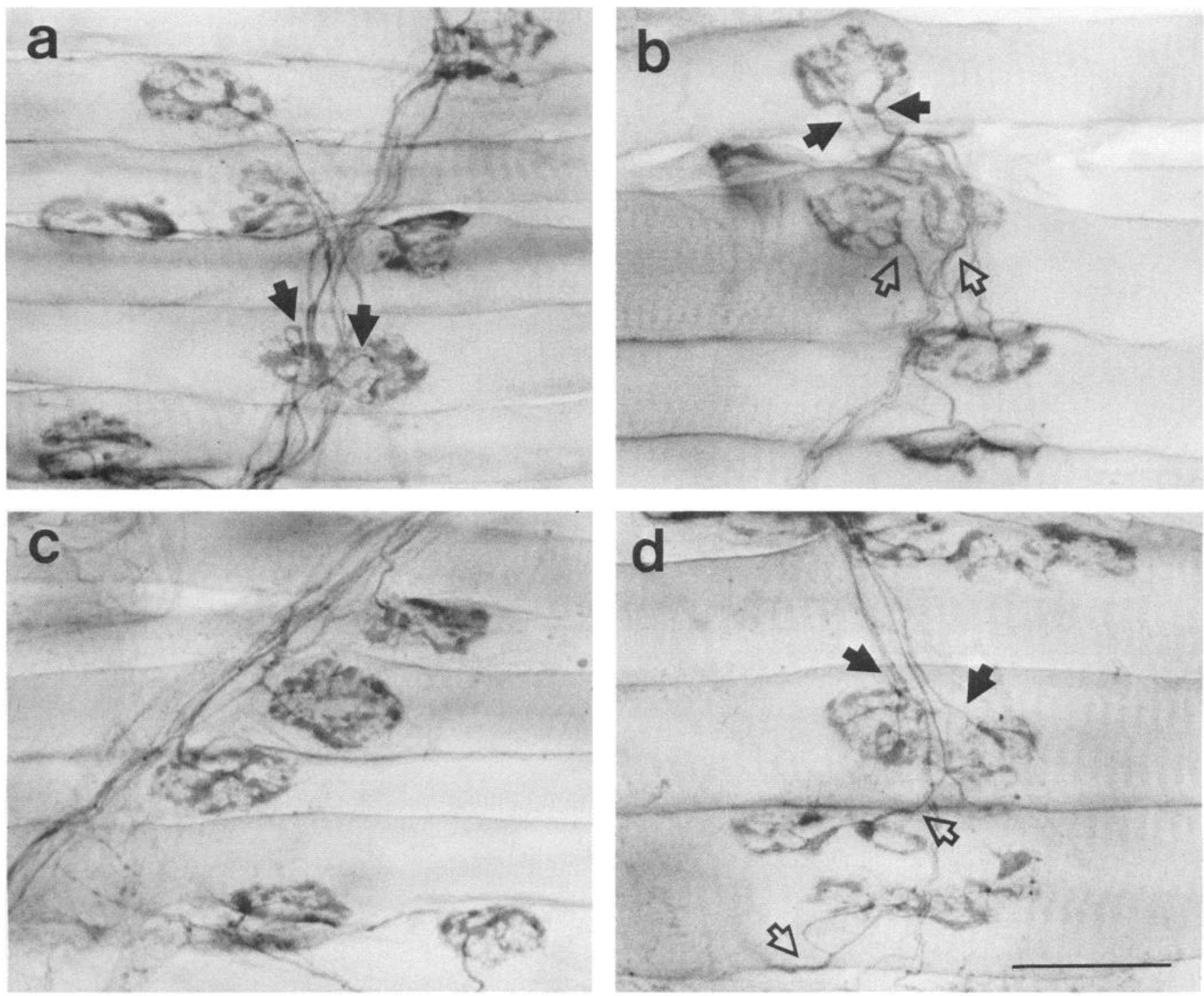

Figure 5. Examples of the morphology of neuromuscular innervation in TNBT stained LA muscles from oil-treated ( $a, c)$ and androgen-treated $(b, d)$ castrates at P21 $(a, b)$ and P28 $(c, d)$. Multiple innervation can be of 2 morphological types, involving either single or multiple junctional sites. Solid arrows point to different axons that multiply innervate a fiber at single junctional sites. Open arrows point to different axons that multiply innervate a fiber at separate junctional sites. In $b, 2$ different axons ( filled arrows) contact the fiber at a single junctional site; one axon branches preterminally (branch point indicated by right arrow) before contacting the fiber. The fiber below in $b$ is also innervated by 2 different axons (the open arrow on the left points to an axon that descends from above the fiber, whereas the open arrow on the right ascends from below the fiber), but in this case, axons contact the fiber at separate junctional sites; each axon forms distinctly separate terminal arborizations that are similar in size and complexity. Such single and multisite multiple innervation is also shown in $d$. Scale bar, $30 \mu \mathrm{m}$.

of an effect of androgen on singly innervated fibers reflects a ceiling effect, since virtually all fibers that are singly innervated in androgen-treated muscles are also innervated by axons that have branched preterminally. An effect of androgen treatment on singly innervated fibers was revealed when the mean number of preterminal branches per fiber was examined. Androgen treatment increased this measure for singly innervated fibers at P21 $(p<0.005)$ and P28 $(p<0.006$, Table 3$)$, but not for multiply innervated fibers. Preterminal branching in muscles from oiltreated castrates was the same as normal at all ages $(p$ 's $>0.05$, Fig. 7, Table 3).

\section{Terminal sprouts}

Fine axonal growth processes were observed to extend beyond the main body of the terminal arbor and appeared as terminal sprouts or filopodia (Fig. 3). Since the increased level of multiple innervation in androgen-treated muscles at P21 and 28 might be the result of androgen-induced terminal sprouting, the percentage of fibers having terminal sprouts was estimated. We also attempted to quantify the number of filopodia but found that this measure varied significantly with the quality of TNBT staining, and thus, the number of filopodia could not be reliably measured. Terminal sprouts were distinguished from filopodia on the basis of size and shape (Fig. 3). Growth processes were identified as sprouts if they extended at least $4.5 \mu \mathrm{m}$ from the main perimeter of the terminal. However, growth processes were occasionally encountered that were clearly not filopodia because of their well-defined bulbous distal tip but were nevertheless shorter than $4.5 \mu \mathrm{m}$. These were also counted as terminal sprouts, with a minimum length of $2 \mu \mathrm{m}$. These criteria seemed effective 


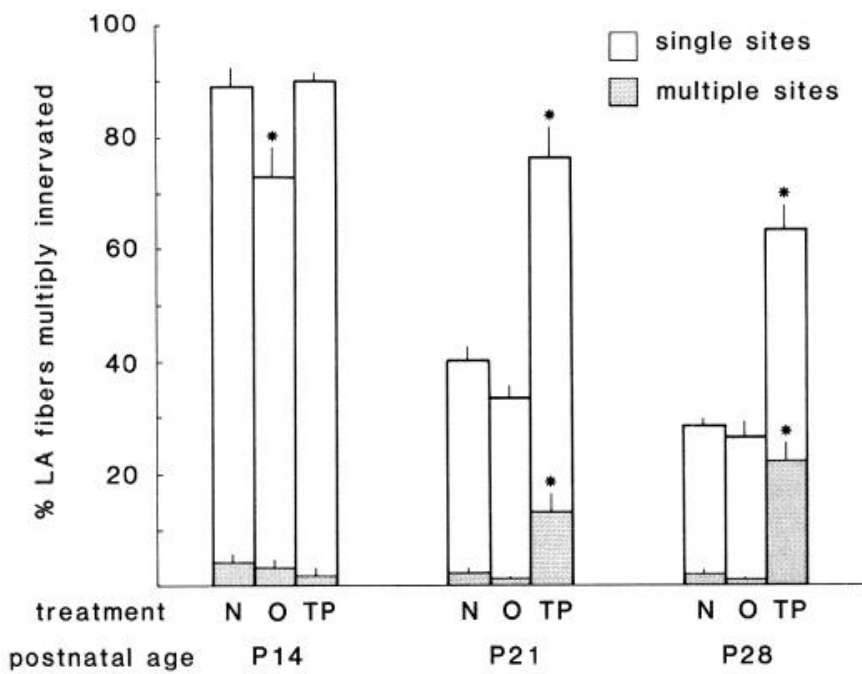

Figure 6. Effect of androgen on the percentage of multiply innervated LA muscle fibers having single or multiple junctional sites. The combined height of open and filled zortions of the bar represents the total mean percentage of multiply innervated muscle fibers in the LA (as in Fig. 2), with the open portion of each bar representing the proportion (mean \pm SEM) of such fibers having single junctional sites and the filled portion of each bar representing the proportion (mean \pm SEM) of such fibers having multiple junctional sites. (The morphology of such single and multisite multiple innervation is shown in Fig. 5.) Treatment groups are defined as in Figure 4. Androgen treatment induces the formation of multiple junctional sites, since androgen-treated muscles at P21 and P28 have significantly more of this type of multiple innervation than normal. However, androgen-treated muscles also have significantly more fibers that are multiply innervated by virtue of contacts formed at a single site.

and reliable in discriminating filopodia from sprouts on the basis of their known morphological characteristics (Hoffman, 1950; Letourneau, 1982).

Terminal sprouts were generally short (Table 4), unbranched, and remained on the fiber of origin. They were never seen to innervate other fibers. Terminal sprouts generally occurred on less than $10 \%$ of LA fibers (Table 4$)$. Their frequency increased significantly between P14 and P21 $(p<0.001)$ but not between P21 and $28(p>0.05)$. Only at P21 did treatment also significantly influence the level of terminal sprouting $(p<0.001)$. Androgen appeared to suppress terminal sprouting, since terminal sprouts were least frequent in muscles from androgentreated castrates and most frequent in muscles from oil-treated castrates (Table 4).

\section{Development of adult motor terminal morphology}

It is well known that during synapse elimination, motor terminals develop what is essentially their adult morphology (Ny-

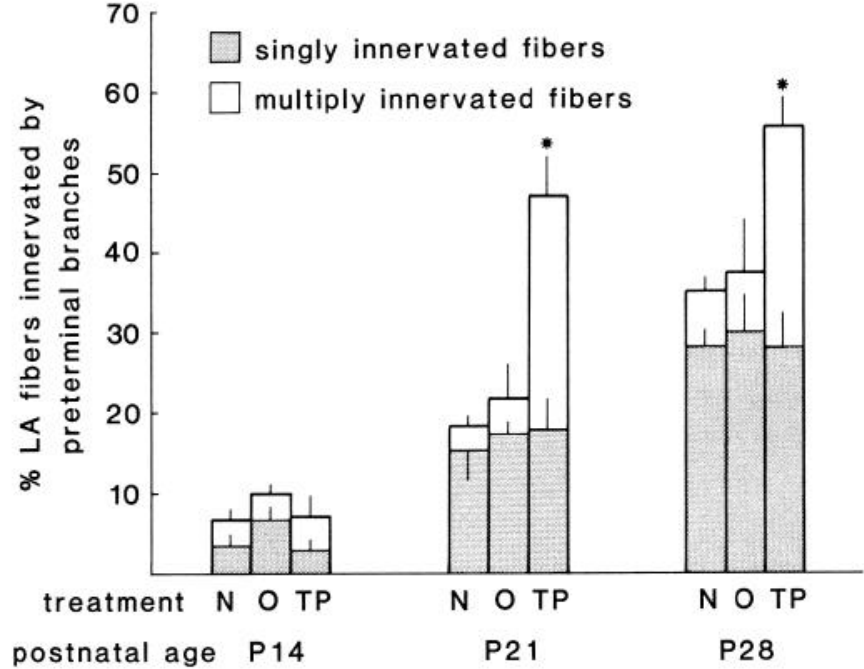

Figure 7. Effect of androgen on the percentage of LA muscle fibers that are innervated by preterminal branches from the same motor axon (such preterminal branching is shown in Fig. 5, $b, d$ ). The combined height of the open and filled portions of the bars represents the total mean percentage of LA fibers innervated by preterminal branches, which is based on muscles from 5-7 animals per group. The open portion of each bar represents those fibers that are singly innervated $( \pm$ SEM) and the filled portion of each bar represents those fibers that are multiply innervated $( \pm$ SEM $)$. Androgen increased only the percentage of multiply innervated fibers with preterminal branching.

strom, 1968; Letinsky and Morrison-Graham, 1980; Steinbach, 1981; Slater, 1982). However, there is little known about the detailed morphology of mammalian motor terminals (except at the electron microscopic level) before the stage at which the adult form of the terminal arbor has emerged. Based on our observations of developing motor terminals stained with TNBT, we reported that at early stages of synapse elimination (P14), motor terminals in the LA often have growth cone morphologies (Jordan et al., 1988). Although motor terminals at this stage clearly lack a differentiated terminal arbor, their morphology is nonetheless surprisingly complex, involving numerous filopodia that radiate out from expanded and simpler regions of axonal contact. As motor nerve terminals elaborate branches to develop the differentiated form of the adult arbor, filopodia become shorter and less numerous, giving rise to the more circumscribed appearance of adult motor terminals. This morphological differentiation of LA motor terminals occurs concomitant with the loss of multiple inputs (Jordan et al., 1988).

The morphology of LA motor terminals in muscles from androgen- and oil-treated castrates looked surprisingly normal (other than the apparent segregation of axonal contacts on some fibers), despite marked differences in the extent of multiple in-

Table 3. Mean number of preterminal branches on singly and multiply innervated LA muscle fibers

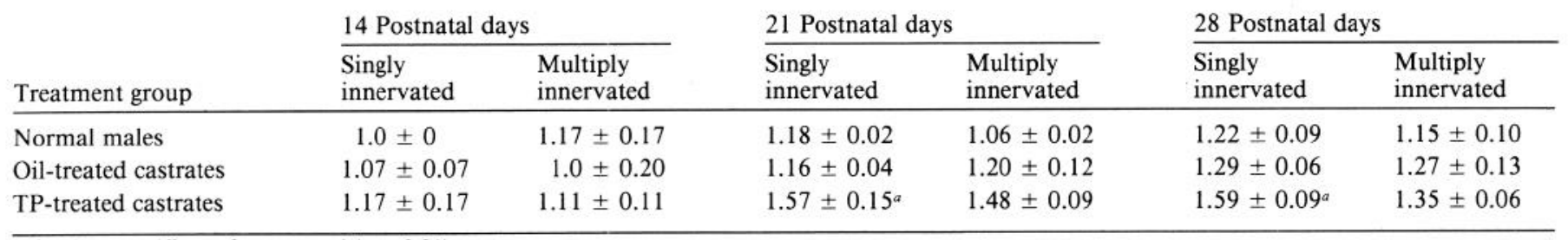

${ }^{a}$ Significantly different from normal $(p<0.01)$. 
Table 4. Percentage of LA muscle fibers having terminal sprouts

\begin{tabular}{|c|c|c|c|c|c|c|}
\hline \multirow[b]{2}{*}{ Treatment group } & \multicolumn{2}{|l|}{14 Postnatal days } & \multicolumn{2}{|l|}{21 Postnatal days } & \multicolumn{2}{|c|}{28 Postnatal days } \\
\hline & $\begin{array}{l}\text { \% fibers with } \\
\text { terminal sprouts }\end{array}$ & $\begin{array}{l}\text { Mean length } \\
(\mu \mathrm{m}) \text { terminal } \\
\text { sprouts }\end{array}$ & $\begin{array}{l}\% \text { fibers with } \\
\text { terminal sprouts }\end{array}$ & $\begin{array}{l}\text { Mean length } \\
(\mu \mathrm{m}) \text { terminal } \\
\text { sprouts }\end{array}$ & $\begin{array}{l}\% \text { fibers with } \\
\text { terminal sprouts }\end{array}$ & $\begin{array}{l}\text { Mean length } \\
(\mu \mathrm{m}) \text { terminal } \\
\text { sprouts }\end{array}$ \\
\hline Oil-treated castrates & $0.5 \pm 0.5$ & $2.3 \pm 0$ & $18.0 \pm 3.6^{a}$ & $6.6 \pm 0.3$ & $10.3 \pm 1.9$ & $6.5 \pm 0.5$ \\
\hline TP-treated castrates & $0 \pm 0$ & $0 \pm 0$ & $2.8 \pm 0.6$ & $4.5 \pm 1.4$ & $4.2 \pm 1.2$ & $5.0 \pm 0.7$ \\
\hline
\end{tabular}

${ }^{a}$ Significantly different from normal $(p<0.013)$.

nervation at P21 and 28 (Fig. 3). Although the level of multiple innervation remained high in androgen-treated muscles, LA motor terminals continued to differentiate, developing an arborization that conformed to the basic adult shape of motor nerve terminals. This observation suggests that the cues controlling the development of adult motor terminal morphology are different from those that control the number of axons that innervate a muscle fiber. Moreover, motor terminals in androgen-treated muscles at P21 and 28 appeared circumscribed and comparable in their complexity to what was observed in LA muscles from oil-treated castrates and normal males of the same age. Most striking was the fact that at P21, when androgen was first seen to induce the formation of separate junctional sites, the morphology of such junctions on single fibers appeared to be at a comparable stage of differentiation. Each possessed adultlike nerve terminal arbors which appeared markedly similar in their complexity (Fig. 5b). At P28, the morphology of LA motor terminals at different sites on the same fiber also appeared comparable in androgen-treated muscles (Fig. $5 d$ ).

\section{Discussion}

The main objective of this study was to determine the effect of androgen on the development of single innervation in the LA and EDL muscles. We found that both endogenous and exogenous androgen delayed the loss of multiple innervation in the LA muscle but not in the EDL muscle. A high level of multiple innervation was maintained in the androgen-treated LA muscle, whereas LA muscles from oil-treated castrates that lacked gonadal androgen had less multiple innervation than normal at P14. Moreover, the excess amount of multiple innervation in androgen-treated LA muscles at P21 and 28 was not formed by terminal sprouts. Three other morphological effects of androgen were to (1) induce the formation of multiple but closely spaced junctional sites on some LA fibers, (2) enhance the development of preterminal branching, and (3) incrcasc the growth of LA muscle fibers.

\section{Androgen and the development of single innervation}

The LA normally undergoes a period of rapid synapse elimination between 2 and 4 weeks after birth. During this period, the majority of LA muscle fibers lose their multiple inputs to become singly innervated. Androgen treatment of castrates prevented much of this loss, whereas castration without androgen treatment hastened the loss of multiple innervation (Fig. 2). It is not clear why castration should produce an early (P14) acceleration of the loss of multiple innervation but not reduce the levels of multiple innervation seen at $\mathrm{P} 21$ and 28. One possibility is that the adrenal glands in these castrated animals increase their synthesis and release of androgen so that castration produces only a transient reduction in androgen levels. Clearly, both endogenous and exogenous androgen after the first week of postnatal life delay the normal development of single innervation in the LA muscle, suggesting that androgen may be capable of regulating mechanisms involved in neuromuscular synapse elimination. Androgen did not influence the normal development of single innervation in the EDL, suggesting that androgen may be capable of influencing synapse elimination in only so-called androgen-sensitive muscles (Jordan et al., 1988). However, the androgen treatment may have simply been initiated too late to prevent the loss of synapses in the EDL since synapse elimination occurs earlier in the EDL than in the LA. This possibility is unlikely because sex differences in androgen secretion (Döhler and Wuttke, 1975; Weisz and Ward, 1980) would otherwise lead to widespread sex differences in neuromuscular synapse elimination, which have not been reported.

\section{Possible cellular processes influenced by androgen to maintain multiple innervation}

There are a number of mechanisms by which androgen might delay the loss of multiple innervation. Androgen may have (1) prevented motoneuronal cell death, (2) stimulated I A muscle cell death, (3) induced the formation of new synapses through terminal and/or collateral sprouting, and/or (4) prevented synapse elimination. It is unlikely that androgen prevents the loss of multiple innervation in the LA by decreasing motoneuron cell death. The LA is innervated by motoneurons located in the spinal nucleus of the bulbocavernosus (SNB) (Breedlove and Arnold, 1980). Although the number of SNB motoneurons can be controlled by androgen through a regulation of motoneuronal cell death, the critical period for this effect ends before P7 (Breedlove and Arnold, 1983; Breedlove, 1984; Nordeen et al., 1985), and thus before the androgen treatment was given in the present study. Furthermore, the increased level of multiple innervation in androgen-treated LA probably does not result from a loss of LA fibcrs, which might also lcad to an increased convergence of inputs, since androgen promotes the survival of LA fibers, not their degeneration (Cihak et al., 1970; Breedlove and Arnold, 1983). It is also clear that androgen does not maintain a higher than normal level of multiple innervation by inducing the formation of synapses through terminal sprouts. The incidence of sprouted terminals was highest in muscles that lacked gonadal androgen and lowest in androgen-treated muscles (Table 4), indicating that androgen actually suppressed this form of sprouting in the LA. In addition, terminal sprouts were never observed to innervate other fibers.

It is less clear whether the increased level of multiple innervation in androgen-treated muscles is the result of androgeninduced axon collateral sprouting since this sort of sprouting may be difficult to detect in a fully innervated muscle, particularly if sprouted axons come to innervate what were already 
innervated junctions. One possible scenario is that androgen induces axons to sprout new collateral branches that then grow to establish new junctional sites on already innervated muscle fibers. Such sprouting might indeed give rise to the multiple junctional sites that emerge at the same time that androgen prevents the loss of multiple innervation (Fig. 6). However, this scenario seems unlikely. In partially denervated adult muscle, recently sprouted axons and their synaptic contacts are recognizable on the basis of their morphology (Hoffman, 1950). Such newly established innervation is formed by smaller-caliber axons and motor terminals that have distinctly simpler morphologies. If multiple junctional sites are formed through axon collateral sprouting, such that new synaptic contacts are added beside older ones, then one might expect to see some degree of heterogeneity in the morphology of axonal contacts at different sites on the same fiber. Some of the junctions might have simpler morphologies and finer-caliber axons, whereas other junctions might have more mature (larger-caliber) axons and contacts. Furthermore, since the morphology of LA motor terminals changes dramatically, progressing from a growth cone morphology at P14 to the differentiated adult form by P21 (Jordan et al., 1988), such newly formed contacts might be distinct in having the less mature growth cone morphology. None of these heterogeneous terminal morphologies was observed. Synaptic contacts at different junctional sites on a single fiber appeared comparably developed and had similarly sized axons and motor terminals (Fig. 5). The growth cone morphologies typical of LA motor terminals at P14 were never seen in LA muscles at P21 and 28 , including those fibers having innervation at separate junctional sites. These observations therefore suggest that the development of multiple junctional sites does not represent the formation of new synapses through androgen-induced collateral sprouting. Moreover, motor axons and their terminals have consistently been reported to have a diminished capacity to sprout during the period of synapse elimination (Thompson and Jansen, 1977; Brown et al., 1981; Caldwell and Ridge, 1983), making it unlikely that motor axons in the LA have sprouted to form new synaptic contacts. Rather, the formation of multiple junctional sites may represent the adult remnants of 2 or more growth cones that were maintained as a result of androgen, yet grew away from each other along the fiber membrane during the period when motor terminals develop their adult arborization (i.e., during synapse elimination). It is important to note that the distance between such separate terminal arbors was often only a few microns (Table 2).

While we lack evidence suggesting that multiple junctional sites develop through an androgenic induction of sprouting, it is also noteworthy that the majority of multiply innervated fibers in androgen-treated LA muscles at P2 1 and 28 were innervated at single junctional sites and, therefore, appeared quite normal (Fig. 6). Moreover, androgen-treated muscles had significantly more of the single-site multiple innervation than normal. Thus, our data suggest that androgen maintains multiple innervation in the LA muscle by preventing the normal ontogenetic process of synapse elimination. It further suggests that androgen prevents the loss of polyneuronal innervation. Indeed, preliminary evidence based on intracellular recording of endplate potentials indicates that LA muscles exposed to androgen treatment during synapse elimination contain more polyneuronal innervation than normal (Jordan et al., 1989).

It is significant that neuromuscular inactivity during synapse elimination in other rat muscles produces effects similar to those produced by androgen (Thompson, 1985). Inactivity delays the development of single innervation and induces the formation of multiple junctional sites (Caldwell and Ridge, 1983). This suggests that androgen may act to influence synapse elimination in the LA by regulating, directly or indirectly, some aspect of its activity. For example, androgen treatment also increased the size (width) of LA fibers (Fig. 1). If such an effect is not paralleled by an increase in the size and/or release properties of LA motor terminals, then LA fibers in androgen-treated muscles might contract less often. However, it is also possible that androgen and activity act through entirely independent means to influence some common underlying mechanism(s) involved in the control of synapse elimination.

\section{Androgen treatment and the development of multiple junctional sites}

The rules involved in the androgenic induction of multiple junctional sites are not simple. Multiple junctional sites developed in androgen-treated LA muscles during the period when multiple innervation is normally eliminated (Fig. 6). Androgen induced the formation of only closely spaced junctions and not the more distantly spaced junctions that normally occur on a small number of LA fibers (Table 2). Androgen induced the formation of multiple junctional sites on only some multiply innervated fibers; most fibers innervated by multiple axons in androgen-treated LA muscles possessed only single junctional sites (Fig. 6). On those fibers with multiple junctional sites, individual sites were sometimes multiply innervated. Finally, such multiple junctional sites also developed on a minority of singly innervated fibers.

Because multiple junctional sites are known to develop during reinnervation of muscle fibers and are sometimes formed by branches of a single axon (Brown et al., 1976; Kuffler et al., 1980), it seems that the simplest explanation for the development of multiple junctional sites is that androgen has induced some axons to sprout collateral branches and form synapses on fibers that they already innervate. Indeed, such a process may normally occur in frog muscle (Nudell and Grinnell, 1983). This possibility is consistent with our observations that androgen increased both the incidence of multiple junctional sites and preterminal branching on singly innervated fibers. However, this possibility is unlikely for reasons already discussed. Clearly, other techniques are needed to determine whether the development of multiple junctional sites on some multiply innervated fibers is associated with branches of the same motor axon and therefore represents the formation of new synaptic contacts through androgen-induced collateral sprouting.

\section{Androgen treatment and the development of preterminal branching}

As multiple innervation is eliminated (through P28), LA axons develop their preterminal branches (Fig. 7) (Jordan et al., 1988). Androgen treatment during this period further increased the number and extent of preterminal branching in the LA (Fig. 7, Table 3). There does not appear to be any strict correlation between the apparent maintenance of multiple innervation and the induction of preterminal branching by androgen. For example, at P28, androgen-treated muscles had more fibers innervated by multiple, unbranched axons than normal muscles (36 vs $21 \%$ ). This suggests that androgen maintains multiple innervation on some fibers without inducing the formation of preterminal branches. It has been suggested that preterminal 
branching represents a means for the elaboration and growth of motor nerve terminals as muscle fibers grow (Tuffery, 1971; Tweedle and Stephens, 1981; Rotshenker and Tal, 1985). Since androgen also increased the growth of LA muscle fibers (Fig. 1), the induction of preterminal branching by androgen may similarly represent a means for accentuated growth and elaboration of LA motor terminals.

\section{Androgen and muscle fiber growth}

The size (width) of adult LA fibers depends on gonadal androgen (Venable, 1966). Following castration of adults, LA fibers atrophy and androgen replacement therapy can reverse the effect of castration. The size of LA fibers during juvenile development is similarly influenced by gonadal androgen (Fig. 1). Besides maintaining multiple innervation, androgen also increased the size of LA fibers. In the mature Xenopus pectoralis muscle, which contains both singly and multiply innervated fibers, multiply innervated fibers tend to be larger than singly innervated ones (Nudell and Grinnell, 1983). On the basis of this relationship, it was suggested that fiber size may be an important factor in determining the number of inputs to a fiber (Nudell and Grinnell, 1983). In short, fibers grow and thereby require or are able to maintain more innervation. This idea is supported by thc obscrvation that androgen-treated LA muscles have larger fibers and more multiple innervation than normal (except at P14), but this relationship breaks down when oil-treated LA muscles are compared with normal. At P14, oil-treated castrates have the same size LA fibers as normal muscles but less multiple innervation. At P21, there is the opposite relationship. This suggests that androgen may regulate the amount of multiple innervation in the LA independent of its effect on muscle fiber diameter.

\section{Androgen and possible mechanisms involved in synapse elimination}

While androgen treatment prevented many LA fibers from becoming singly innervated, it is of interest that some amount of multiple innervation was lost even in androgen-treated muscle. There are several possible explanations for this. The most likely possibility is that only some mechanisms involved in synapse elimination are sensitive to androgenic regulation. Multiple mechanisms that involve both central and peripheral processes may operate to establish the adult pattern of synaptic connections in muscle (Brown et al., 1976; Thompson and Jansen, 1977). Evidence suggests that the loss of neuromuscular connections reflects, in part, some tendency for motoneurons to retract their axonal arbor. This tendency may involve metabolic or other processes located centrally that limit the number of axon terminals a motoneuron can maintain. It is also clear that peripheral competitive interactions are involved since experimentally reducing the amount of convergence among motor axons during synapse elimination results in motoneurons maintaining more synapses than normal (Brown et al., 1976; Thompson and Jansen, 1977; Betz et al., 1980). One possible scenario is that androgen rescues some LA synapses by increasing the supply of some muscle-produced trophic substance for which motor terminals may compete during the elimination process (Jansen et al., 1978; Purves, 1986). However, this effect may not fully compensate for other androgen-insensitive mechanisms that drive the elimination process. Since SNB motoneurons and the LA muscle in adult rats both possess androgen receptors (Dionne et al., 1979; Breedlove and Arnold, 1980), each represents a candidate site for androgen action during synapse elimination. By restricting androgen exposure to either the lumbar spinal cord containing SNB motoneurons or to the LA, it should be possible to answer whether androgen maintains multiple innervation in the LA by regulating central and/or peripheral mechanisms involved in synapse elimination. Other possible explanations for the partial sparing of synapses by androgen are that a complete sparing of synapses might require a higher dose of androgen or might be due to only some motoneurons and/or muscle fibers having androgen receptors.

The data from this study raise the question of whether synapse elimination will occur in the LA once androgen treatment has ended. In a companion paper (Jordan et al., 1989), we report that the LA retains a high level of multiple innervation for at least 2 months after the end of juvenile androgen treatment, suggesting that androgen during the period of synapse elimination can irreversibly determine the adult pattern of innervation in the LA and probably does so by permanently preventing synapse elimination.

\section{References}

Balice-Gordon, R. J., and W. J. Thompson (1988) Synaptic rearrangements and alterations in motor unit properties in neonatal rat extensor digitorum longus muscle. J. Physiol. (Lond.) 398: 191-210.

Bennett, M. R., and A. G. Pettigrew (1974) The formation of synapses in striated muscle during development. J. Physiol. (Lond.) 241: 514 545.

Betz, W. J., J. H. Caldwell, and R. R. Ribchester (1979) The size of motor units during postnatal development of rat lumbrical muscle. J. Physiol. (Lond.) 297: 463-478.

Betz, W. J., J. H. Caldwell, and R. R. Ribchester (1980) The effects of partial denervation at birth on the development of muscle fibres and motor units in the rat lumbrical muscle. J. Physiol. (Lond.) 303: 265-279.

Breedlove, S. M. (1984) Androgen forms sexually dimorphic spinal nucleus by saving motoneurons from programmed death. Soc. Neurosci. Abstr. 10:927.

Breedlove, S. M., and A. P. Arnold (1980) Hormone accumulation in a sexually dimorphic motor nucleus of the rat spinal cord. Science 210: 564-566.

Breedlove, S. M., and A. P. Arnold (1983) Hormonal control of a developing neuromuscular system: II. Sensitive periods for the androgen induced masculinization of the rat spinal nucleus of the bulbocavernosus. J. Neurosci. 3: 424-432.

Brown, M. C., J. K. S. Jansen, and D. Van Essen (1976) Polyneuronal innervation of skeletal muscle in new-born rats and its elimination during maturation. J. Physiol. (Lond.) 261: 387-422.

Brown, M. C., R. L. Holland, and W. G. Hopkins (1981) Restoration of focal multiple innervation in rat muscles by transmission block during a critical stage of development. J. Physiol. (Lond.) 318: 355364.

Buresova, M., and E. Gutmann (1971) Effect of testosterone on protein synthesis and contractility of the levator ani muscle of the rat. $\mathrm{J}$. Endocrinol. 50: 643-651.

Caldwell, J. H., and M. A. P. Ridge (1983) The effects of deafferentation and spinal cord transection on synapse elimination in developing rat muscles. J. Physiol. (Lond.) 339: 145-159.

Cihak, R., E. Gutmann, and V. Hanzlikova (1970) Involution and hormone-induced persistence of the muscle sphincter (levator) ani in female rats. J. Anat. 106: 93-110.

Corpechot, C., E. E. Baulieu, and P. Robel (1981) Testosterone, dihydrotestosterone and androstanediols in plasma, testes and prostates of rats during development. Acta Endocrinol. 96: 127-135.

Dennis, M. J., L. Ziskind-Conhaim, and A. J. Harris (1981) Development of neuromuscular junctions in rat embryos. Dev. Biol. 81 : 266-279.

Dionne, F. T., J. Y. Dube, R. L. Lesage, and R. R. Tremblay (1979) In vivo androgen binding in rat skeletal and perineal muscles. Acta Endocrinol. 91: 362-372.

Döhler, K. D., and W. Wuttke (1975) Changes with age in levels of 
serum gonadotropins, prolactin and gonadal steroids in prepubertal male and female rats. Endocrinology 97: 898-907.

Hoffman, H. (1950) Local re-innervation in partially denervated muscle: A histo-physiological study. Aust. J. Exp. Biol. 28: 383-397.

Jansen, J. K. S., W. Thompson, and D. P. Kuffler (1978) The formation and maintenance of synaptic connections as illustrated by studies of the neuromuscular junction. Prog. Brain Res. 48: 3-18.

Jordan, C. L., M. S. Letinsky, and A. P. Arnold (1988) Synapse elimination occurs late in the hormone-sensitive levator ani muscle of the rat. J. Neurobiol. 19: 335-356.

Jordan, C., M. S. Letinsky, and A. P. Arnold (1989) The role of gonadal hormones in neuromuscular synapse elimination in rats: II. Multiple innervation persists in the adult levator ani after juvenile androgen treatment. J. Neurosci. 9: 239-247.

Keppel, G. (1982) Design and Analysis, Prentice-Hall, Englewood Cliffs, NJ.

Kuffler, D. P., W. Thompson, and J. K. S. Jansen (1980) The fate of foreign endplates in cross-innervated rat soleus muscle. Proc. R. Soc. Lond. [Biol.] 208: 189-222.

Letinsky, M. S., and K. Morrison-Graham (1980) Structure of developing frog neuromuscular junctions. J. Neurocytol. 9: 321-342.

Letourneau, P. C. (1982) Nerve fiber growth and its regulation by extrinsic factors. In Neuronal Development, N. C. Spitzer, ed., pp. 213-254, Plenum, New York.

Nordeen, E. J., K. W. Nordeen, D. R. Sengelaub, and A. P. Arnold (1985) Androgens prevent normally occurring cell death in a sexually dimorphic spinal nucleus. Science 229: 671-673.

Nudell, B. M., and A. D. Grinnell (1983) Regulation of synaptic position, size, and strength in anuran skeletal muscle. J. Neurosci. 3 . 161-176.

Nystrom, B. (1968) Postnatal development of motor nerve terminals in "slow-red" and "fast-white" cat muscles. Acta Neurol. Scand. 44: 363-383.

Purves, D. (1986) The trophic theory of neural connections. TINS 9: 486-489.

Riley, D. A. (1977) Spontaneous elimination of nerve terminals from the endplates of developing skeletal myofibers. Brain Res. 134: 279285.

Riley, D. A. (1981) Ultrastructural evidence for axon retraction during the spontaneous elimination of polyneuronal innervation of the rat soleus muscle. J. Neurocytol. 10:425-440.

Rotshenker, S., and D. Tal (1985) The transneuronal induction of sprouting and synapse formation in intact mouse muscle. J. Physiol. (Lond.) 360: 387-396.

Slater, C. R. (1982) Postnatal maturation of nerve-muscle junctions in hindlimb muscles of the mouse. Dev. Biol. 94: 11-22.

Smith, E. R., D. A. Damassa, and J. M. Davidson (1978) Hormone administration: Peripheral and intracranial implants. Methods Psychobiol. 3: 259-279.

Souccar, C., A. J. Lapa, and J. R. DoValle (1982) Influence of castration on the electrical excitability and contraction properties of the rat levator ani muscle. Exp. Neurol. 75: 576-588.

Steinbach, J. H. (1981) Developmental changes in acetylcholine receptor aggregates at rat skeletal neuromuscular junctions. Dev. Biol. 84: $267-276$.

Thompson, W. (1985) Activity and synapse elimination at the neuromuscular junction. Cell. Mol. Neurobiol. 5: 167-182.

Thompson, W. J. (1986) Changes in the innervation of mammalian skeletal muscle fibers during postnatal development. TINS 9: 25-28.

Thompson, W., and J. K. S. Jansen (1977) The extent of sprouting of remaining motor units in partly denervated immature and adult rat soleus muscle. Neuroscience 2: 523-535.

Tucek, S., D. Kostirova, and E. Gutmann (1976) Testosterone-induced changes of choline acetyl-transferase and cholinesterase activities in rat levator ani muscle. J. Neurol. Sci. 27: 353-362.

Tuffery, A. R. (1971) Growth and degeneration of motor end-plates in normal cat hind limb muscles. J. Anat. 110: 221-247.

Tweedle, C. D., and K. E. Stephens (1981) Development of complexity in motor nerve endings at the rat neuromuscular junction. Neuroscience 6: 1657-1662.

Van Essen, D. C. (1982) Neuromuscular synapse elimination. In Neuronal Development, N. C. Spitzer, ed., pp. 333-376, Plenum, New York.

Venable, J. H. (1966) Morphology of the cells of normal, testosteronedeprived, and testosterone-stimulated levator ani muscles. Am. J. Anat. 119: 271-302.

Vyskocil, F., and E. Gutmann (1977) Anabolic effects of testosterone on the levator ani muscle of the rat. Pfluegers Arch. 371:3-8.

Wainman, P., and G. C. Shipounoff (1941) The effects of castration and testosterone propionate on the striated perineal musculature in the rat. Endocrinology 29: 975-978.

Weisz, J., and I. L. Ward (1980) Plasma testosterone and progesterone titers of pregnant rats, their male and female fetuses and neonatal offspring. Endocrinology 106: 306-316. 